This is the accepted version of the article:

Yazdi A., Merkoçi F., Bastús N., I maz I., Puntes V., Maspoch D.. The influence of the MOF shell thickness on the catalytic performance of composites made of inorganic (hollow) nanoparticles encapsulated into MOFs. Catalysis Science \& Technology, (2016). 6. : 8388 - . 10.1039/C6CY02071B.

Available at: https://dx.doi.org/10.1039/C6CY02071B 
-Received 00th January 20xx Accepted 00th January 20xx

DOI: $10.1039 / \times 0 \times x 00000 x$

\section{The influence of the MOF shell thickness in the catalytic performance of composites made of inorganic (hollow) nanoparticles encapsulated into MOFs}

\author{
A. Yazdia , F. Merkocia , N. Bastus ${ }^{a}$, I. Imaz ${ }^{a}$, V. Puntes ${ }^{a, b, c,{ }^{*}}$ and D. Maspoch ${ }^{a, b, *}$
}

www.rsc.org/chemcomm

Herein we report the encapsulation of hollow $\mathrm{Pt}$ or $\mathrm{Pd}$ nanoparticles into ZIF-8, making a series of composites in which the ZIF-8 shell thickness has been systematically varied. By using these composites as catalysts for the reduction of 4-nitrophenol and Eosin $Y$, we show that the MOF shell thickness plays a key role in the catalytic performance of this class of composites.

The combination of two or more materials forming composites is a powerful strategy to create new materials with superior (multi)functions. With this aim, inorganic nanoparticles (iNPs) have recently been incorporated in metal-organic frameworks (MOFs) to create a new class of porous composites (hereafter called MOF@iNP) with interesting applications in sensing, catalysis, plasmonics and pollutant sequestration and

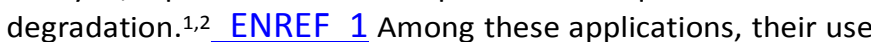
for heterogeneous catalysis is promising. Combining iNPs and MOFs overcomes some of the intrinsic problems of iNPs when used in catalysis, ${ }^{3}$ such as their tendency to aggregate, their low recyclability, and the difficulty to recover them from the reaction media. MOFs can support and protect them, thus facilitating their recovery from the bulk solution and preventing particle aggregation. In addition, the well-defined pore characteristics of MOFs can ideally permit the selective gating of the molecules that reach, and therefore react with the iNPs.

The most common methods to produce MOF@iNP composites include infiltration of the iNP precursors and subsequent growth of the iNPs inside the MOF; 4 attachment of the iNPs onto the surface of the MOF; ${ }^{5}$ and the use of iNPs as nucleation centers to growth the MOF around them, thus encapsulating the iNPs into the synthesized MOFs. First reported by Huo et al., ${ }^{6}$ this latter method is very versatile to design MOF@iNPs for heretogeneous catalysis applications since it allows using and protecting iNPs with no size, shape and nature restriction as well as controlling the number and localization of these iNPs inside the MOFs. For example, analogous ZIF-8@iNP composites have been successfully synthesized with Pt and Au NPs of different sizes (e.g. 2.5, 2.7,
3.3 and $4.1 \mathrm{~nm}$ for Pt; and 13, 15 and $34 \mathrm{~nm}$ for $\mathrm{Au})^{6-8}$, Au NPs of different shapes (spheres and cubes), and iNPs of different nature (e.g. $\mathrm{Au}, \mathrm{Pt}, \mathrm{Fe}_{3} \mathrm{O}_{4}, \mathrm{CdTe}, \mathrm{CdSe}$ and $\mathrm{Pd}$ ). 6,9 Also, ZIF$8 @$ Au composites in which the embedded Au NPs are localized at the core (even at the single particle level) or spread over the ZIF-8 crystals have been generated. ${ }^{9-11}$

To date, some advances have been made in using these MOF@iNP composites for heterogeneous catalysis. For example, ZIF-8@AuNP and ZIF-8@PtNP composites have shown to be efficient catalysts for the oxidation of $\mathrm{CO},{ }^{6}$ the reduction of 4-nitrophenol (4-NP), ${ }^{9,} 12$ the oxidation of benzyl alcohol, ${ }^{11}$ and the hydrogenation of alkynes. ${ }^{7}$ Thus far, however, almost no one has paid special attention to study which parameters play a key role in the catalytic performance of these MOF@iNP composites. Farha et al. noticed that an increase of the external crystal surface of MOF leads to an increase of the yield of the hydrogenation of alkynes. ${ }^{7}$ Here we report on the influence of the MOF shell thickness on the catalytic performance of this class of MOFs@iNP composites. We postulate that this parameter must be critical for their catalytic properties since it must directly impact on the accessibility and diffusion of the molecules to the iNPs. In this study, we chose ZIF- 8 as the MOF, and hollow Pt and Pd NPs as the iNPs. We chose ZIF- 8 because it has been widely used to produce MOF@iNP composites. And we chose hollow Pt and Pd NPs because they present some advantages over their solid counterparts in terms of lighter weight, saving of material, reduction of cost and superior catalytic activities associated to a nanoreactor cage effect and to the highly active uncapped and rough inner surfaces. In fact, they are excellent catalysts for the two model reactions selected for this study: the reduction of 4-NP and the bulkier Eosin $Y(E Y)$. Reduction of 4$N P$ is a common reaction since it is usually used for the remediation of 4-NP; a water pollutant with high toxicity. ${ }^{13}$ Reduction of dye EY is a commonly used model reaction to probe the catalytic activity of iNPs. ${ }^{14}$

\footnotetext{
a. Catalan Institute of Nanoscience and Nanotechnology (ICN2), CSIC and The Barcelona Institute of Science and Technology, Campus UAB, Bellaterra, 08193

Barcelona, Spain, E-mail: victor.puntes@icn2.cat; daniel.maspoch@icn2.cat

b. ICREA, Pg. Lluís Companys 23, 08010 Barcelona, Spain

c. Vall, d'Hebron Institut de Recerca (VHIR), 08035, Barcelona, Spain

† Footnotes relating to the title and/or authors should appear here.

Electronic Supplementary Information (ESI) available: [details of any supplementary information available should be included here]. See
} DOI: $10.1039 / \times 0 \times x 00000 x$ 
This study began with the systematic synthesis of a series of ZIF-8@PtNP and ZIF-8@PdNP composites, which vary in size (hereafter defined as $c=\left(c_{1}+c_{2}\right) / 2$; Fig. 1c, Table 1 , and Experimental Section, ESI + ). In a typical synthesis, a methanolic dispersion containing hollow Pt NPs (diameter: $27.4 \pm 2.0 \mathrm{~nm}$; shell: $2.8 \pm 0.6 \mathrm{~nm}$; Fig. 1 a) or hollow Pd NPs (diameter: $35.6 \pm$ $3.8 \mathrm{~nm}$; shell: $2.4 \pm 0.4 \mathrm{~nm}$; Fig. $1 \mathrm{~b}$ ), which were previously synthesized by galvanic replacement using Ag NPs as sacrificial templates (Experimental Section and Fig. S1-S4, ESI+), ${ }^{15,16}$ were first prepared at a concentration of $0.25 \mathrm{mM}$ and $0.3 \mathrm{mM}$, respectively. Then, $4 \mathrm{~mL}$ of these dispersions were introduced into different $20 \mathrm{~mL}$ methanolic solutions of 2-methylimidazole (2-MiM) (2-MiM concentrations: 6.5, 25, 37.5 and $50 \mathrm{mM}$ for Pt; and 6.25, 25 and $50 \mathrm{mM}$ for Pd). After $2 \mathrm{~min}, 20 \mathrm{~mL}$ of a methanolic solution of $\mathrm{Zn}\left(\mathrm{NO}_{3}\right)_{2} \cdot 6 \mathrm{H}_{2} \mathrm{O}$ (of equal molarity to 2MiM) was added to these mixtures. The final mixtures were allowed to react at room temperature without stirring for $24 \mathrm{~h}$. Finally, each one of the synthesized solids were collected by centrifugation (13200 rcf), washed 2 times with methanol and dried overnight at $60^{\circ} \mathrm{C}$.

High angle annular dark field scanning transmission electron microscopy (HAADF-STEM) demonstrated the encapsulation of both hollow Pt and Pd NPs in the inner core of the ZIF-8 crystals with average diameters $c$ ranging from 197 $\pm 26 \mathrm{~nm}$ to $1832 \pm 270 \mathrm{~nm}$, depending on the concentration of 2-MiM and $\mathrm{Zn}\left(\mathrm{NO}_{3}\right)_{2} \cdot 6 \mathrm{H}_{2} \mathrm{O}$ used (Table 1 and Fig. 2). In addition, they clearly showed that the number of encapsulated hollow Pd and Pt NPs per ZIF-8 crystal also increases when increased the concentration of $2-\mathrm{MiM}$ and $\mathrm{Zn}\left(\mathrm{NO}_{3}\right)_{2} \cdot 6 \mathrm{H}_{2} \mathrm{O}$ in the reaction. Thus, to measure the average MOF shell thickness of each sample, we first measure the distance (hereafter defined as distance a) from the centre of the ZIF-8 crystal to the NP located closer to the crystal surface (Fig. 1c).

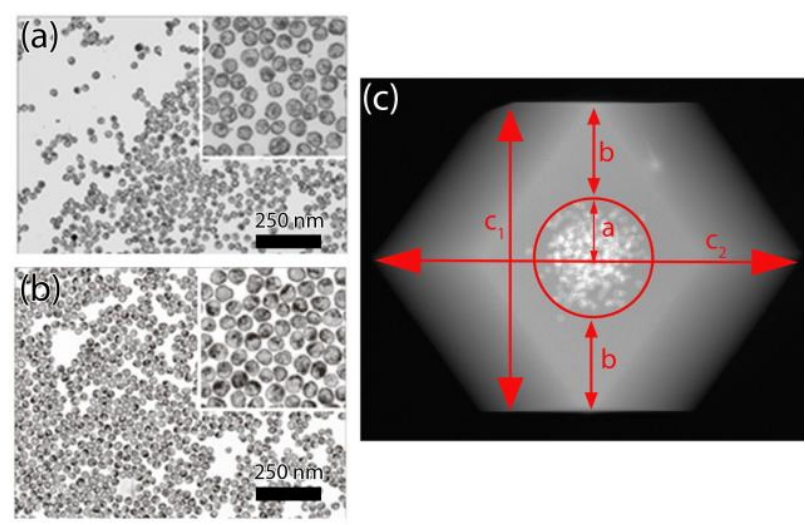

Figure 1. HAADF-STEM images of (a) hollow Pt NPs, (b) hollow Pd NPs, and (c) a single ZIF-8@PdNP composite highlighting the dimensions shown in Table 1.

Table 1. Hollow Pt/Pd NP content and size distribution of the synthesized composites.

\begin{tabular}{ccccc}
\hline Sample & $c(\mathrm{~nm})$ & $b(\mathrm{~nm})$ & $\mathrm{a}(\mathrm{nm})$ & $\begin{array}{c}\text { NPw.t. } \\
(\%)\end{array}$ \\
\hline ZIF-8@PtNP-1 & $284 \pm 54$ & $66 \pm 42$ & $76 \pm 15$ & 2.8 \\
\hline ZIF-8@PtNP-2 & $511 \pm 87$ & $156 \pm 64$ & $99 \pm 21$ & 1 \\
\hline ZIF-8@PtNP-3 & $746 \pm 88$ & $236 \pm 64$ & $137 \pm 20$ & 0.83 \\
\hline ZIF-8@PtNP-4 & $1832 \pm 270$ & $562 \pm 198$ & $354 \pm 63$ & 0.82 \\
\hline ZIF-8@PdNP-1 & $197 \pm 26$ & $55 \pm 24$ & $43 \pm 11$ & 1.76 \\
\hline ZIF-8@PdNP-2 & $453 \pm 70$ & $148 \pm 50$ & $78 \pm 15$ & 0.6 \\
\hline ZIF-8@PdNP-3 & $1689 \pm 152$ & $585 \pm 135$ & $259 \pm 59$ & 0.43 \\
\hline
\end{tabular}
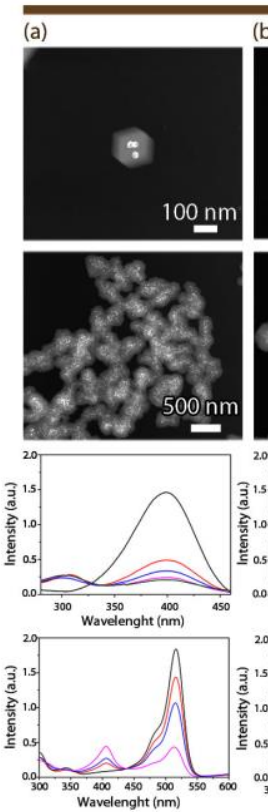
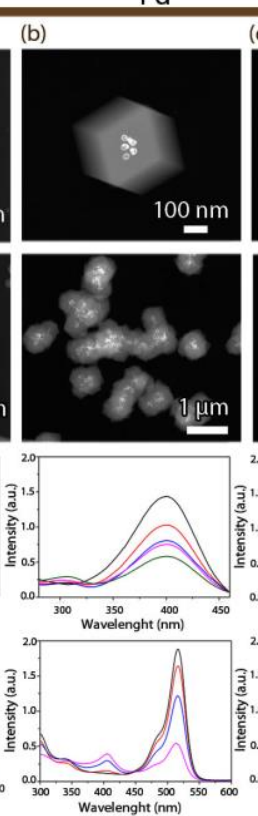
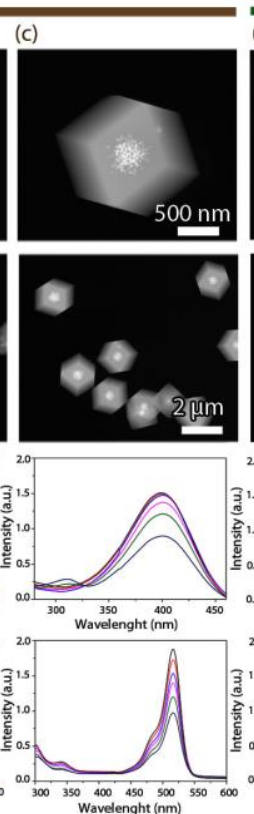
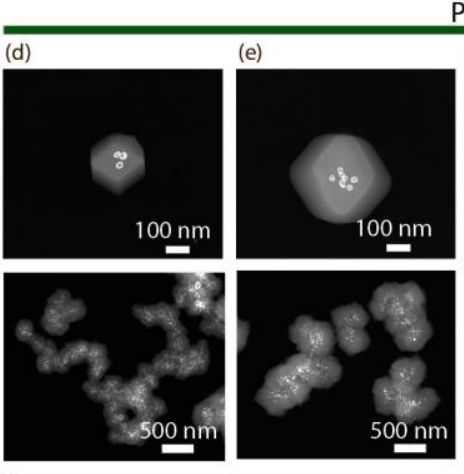

$\mathrm{Pt}$
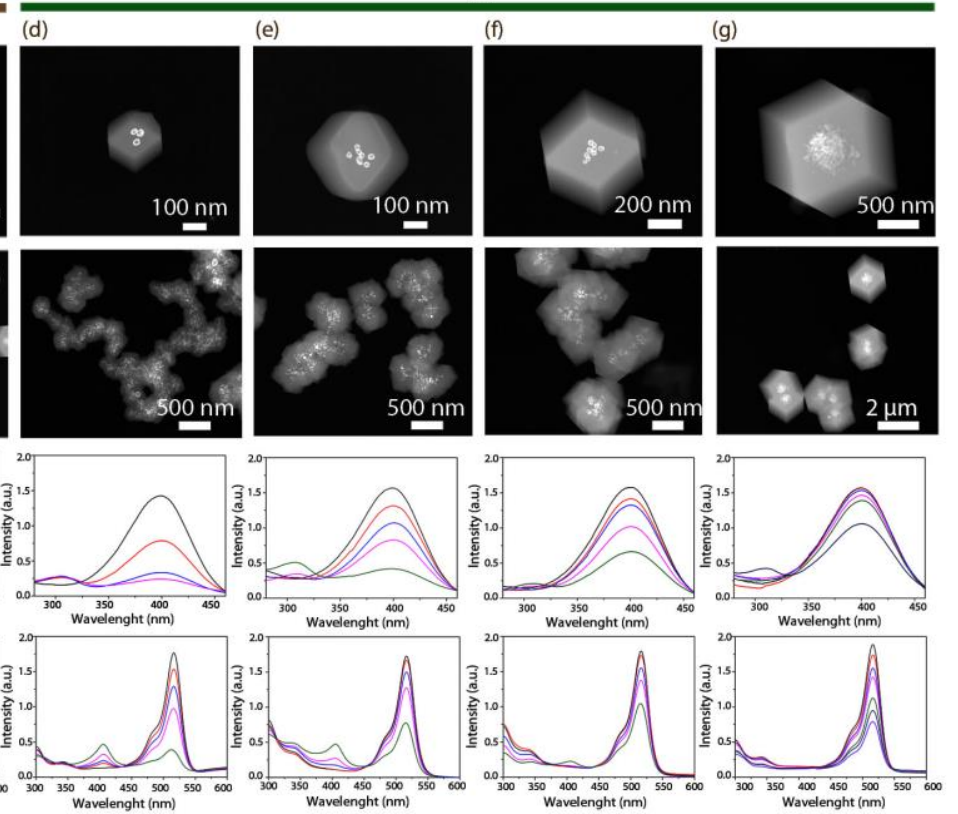

Figure 2. HAADF-STEM images (first row: single composite; second row: general view) of (a) ZIF-8@PdNP-1, (b) ZIF-8@PdNP-2, (c) ZIF-8@PdNP-3, (d) ZIF-8@PtNP-1, (e) ZIF8@PtNP-2, (f) ZIF-8@PtNP-3 and (g) ZIF-8@PtNP-4. Third and forth rows show the UV-Vis evolution of the 4-NP (third row) and EY2- (forth row) reduction using the corresponding composite as catalyst. In these spectra: $0 \mathrm{~min}$ (black); $10 \mathrm{~min}$ (red); $20 \mathrm{~min}$ (blue); $30 \mathrm{~min}$ (pink); $40 \mathrm{~min}$ (green); $50 \mathrm{~min}$ (navy); and $60 \mathrm{~min}$ (violet). 
Then, the average MOF shell thickness $\boldsymbol{b}$ of each composite was obtained from $\boldsymbol{b}=\boldsymbol{c} / \mathbf{2}-\boldsymbol{a}$. In addition, the encapsulation of both hollow NPs was further confirmed by elemental mapping with energy dispersive X-ray spectrometry (EDX) on single composites, which revealed a uniform distribution of $\mathrm{Zn}$ atoms along the composite and the presence of Pt or Pd where the NPs are located (Fig. S5, ESI + ). Finally, the percentage of Pt and $\mathrm{Pd}$ in each composite (\% $\left.\mathrm{W}_{\mathrm{Pd} / \mathrm{Pt}} / \mathrm{w}_{\text {composite }}\right)$ was measured by ICP-MS, whereas the formation of ZIF-8 in all composites was corroborated by powder X-ray diffraction (PXRD; Fig. S6, ESI + ). The different parameters for all synthesized ZIF-8@PtNP and ZIF-8@PdNP composites are listed in Table 1.

To start evaluating the catalytic performance of the synthesized composites, we first studied the aqueous reduction of 4-NP to 4-aminophenol (4-AP) in the presence of $\mathrm{NaBH}_{4}$ and of the composites. In this reaction, $\mathrm{NaBH}_{4}$ is oxidized on the surface of the hollow NPs, provoking the reduction of 4-NP to 4-AP when enters in contact with the NPs. ${ }^{17}$ This reduction can be simply followed by a reduction of the optical absorption at $400 \mathrm{~nm}$ corresponding to 4-NP.

The catalytic reactions were conducted at basic $\mathrm{pH}$ by mixing $0.5 \mathrm{ml}$ of an aqueous solution of $\mathrm{NaBH}_{4}(1.3 \mathrm{M})$ with 3.0 $\mathrm{mL}$ of an aqueous solution of 4-NP $(0.125 \mathrm{mM})$. After $2 \mathrm{~min}$., $0.2 \mathrm{~mL}$ of an aqueous dispersion containing ZIF-8@PtNP-1 at a Pt concentration of $2.8 \% \mathrm{w} / \mathrm{w}$ or ZIF-8@PdNP-1 at a Pd concentration of $1.8 \% \mathrm{w} / \mathrm{w}$ was injected into the reaction mixture, and the reaction was followed every $10 \mathrm{~min}$ by UV-vis spectroscopy in the range of $280-460 \mathrm{~nm}$ (Fig. 2). It is important to highlight here that these reactions were followed only for $30 \mathrm{~min}$ in the case of ZIF-8@PtNP-1 and for $40 \mathrm{~min}$ in the case of ZIF- $8 @ P d N P-1$ because this is the maximum time that the ZIF- 8 shells of the composites remain unaltered, as was confirmed by Scanning Electron Microscopy (SEM; Fig.
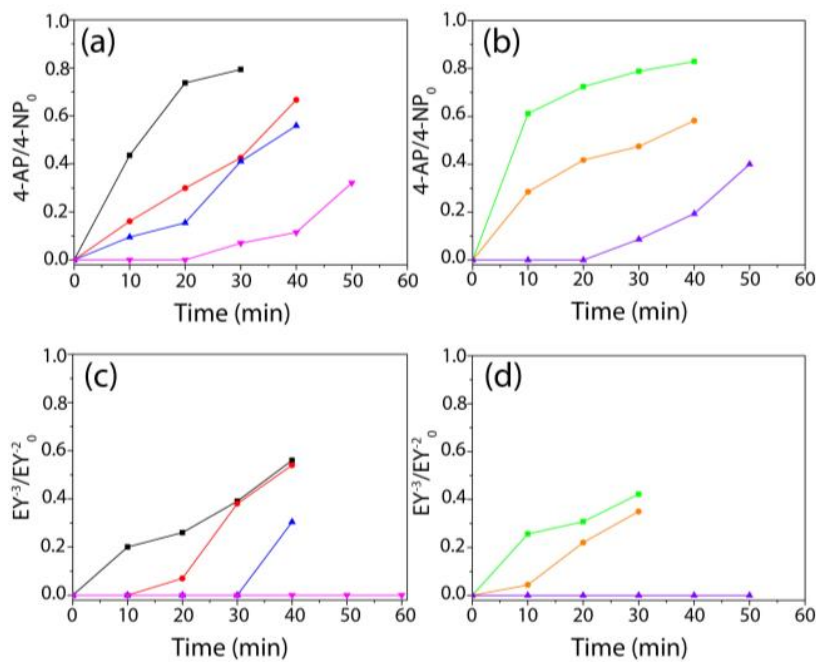

Figure 3. $(\mathrm{a}, \mathrm{b})$ Variation of 4-AP/4-NPo conversion efficiency as a function of time when using (a) ZIF-8@PtNP and (b) ZIF-8@PdNP composites as catalysts. (c,d) Variation of $E Y^{3-} / E Y_{O^{2-}}$ conversion efficiency as a function of time when using (c) ZIF-8@PtNP and (d) ZIF-8@PdNP composites as catalysts. Colour code: ZIF-8@PtNP-1 (black), ZIF-8@PtNP-2 (red), ZIF8@PtNP-3 (blue), ZIF-8@PtNP-4 (pink), ZIF-8@PdNP-1 (green), ZIF8@PdNP-2 (orange) and ZIF-8@PdNP-3 (violet).
S8,S14, ESIt). For longer times, etching of the external ZIF-8 shell was observed, increasing with time and finally exposing the hollow iNPs to the surface of the composites and releasing them. For both catalysts, the concentration of 4-NP at each time was determined using an extinction coefficient of $12.8 \mathrm{x}$ $10^{3} \mathrm{M}^{-1} \cdot \mathrm{cm}^{-1}$ (Fig. S23, ESI + ). The conversion efficiency (4-AP/4$N P_{0}$ ) of each composite was then measured by difference.

Figure 2 shows the evolution of the resulting UV-vis spectra. In both cases, we observed that the intensity of the absorption peak at $400 \mathrm{~nm}$ decreases with time while a new absorption peak at $305 \mathrm{~nm}$ characteristic of 4-AP appears after $10 \mathrm{~min}$ of reaction. The reduction of the peak at $400 \mathrm{~nm}$ also decreased with time, achieving maximum conversion efficiencies of $79 \%$ for the ZIF-8@PtNP-1 and $83 \%$ for the ZIF$8 @ P d N P-1$. These results are important since they confirm that both reactants, 4-NP and $\mathrm{NaBH}_{4}$, are able to cross the ZIF8 shells, with thicknesses of $66 \pm 42 \mathrm{~nm}$ and $55 \pm 24 \mathrm{~nm}$, and reach the catalytic hollow NPs.

To study if the ZIF-8 shell thickness has an effect on the catalytic performance of these composites, we then repeated the same reaction but using the composites with thicker ZIF-8 shells as catalysts. Each reaction was again run for the time that allowed the stability of the ZIF-8 shells (Fig. S8-22, ESI+). Figure $3 a, b$ shows the evolution of the conversion efficiency for the different composites with time. A first clear observation is that, independently of the nature of the iNP, the yield at a specific time decreases as the ZIF- 8 shell thickness increases. For example, at $20 \mathrm{~min}$, the conversion efficiencies were $0 \%$ for both ZIF-8@PtNP-4 and ZIF-8@PdNP-3 with thicknesses of $562 \pm 198 \mathrm{~nm}$ and $585 \pm 135 \mathrm{~nm}$, respectively; $15 \%$ for the ZIF-8@PtNP-3 with a thickness of $236 \pm 64 \mathrm{~nm} ; 30$ $\%$ for the ZIF-8@PtNP-2 with a thickness of $156 \pm 64 \mathrm{~nm} ; 42 \%$ for the ZIF-8@PdNP-2 with a thickness of $148 \pm 50 \mathrm{~nm}$; and 72 $\%$ and $74 \%$ for the ZIF-8@PdNP-1 and ZIF-8@PtNP-1 with thicknesses of $55 \pm 24 \mathrm{~nm}$ and $66 \pm 42 \mathrm{~nm}$, respectively. Another clear evidence from this data is that, independently of the nature of the iNP, the time at which 4-AP is starting to be formed increases as the ZIF- 8 shell thickness also increases. Indeed, for the thicker composites, the formation of 4-AP was detected after $30 \mathrm{~min}$ of reaction.

Both observations agree well with the fact that the diffusion time of 4-NP and $\mathrm{NaBH}_{4}$ molecules through the MOF shell to reach the surface of the hollow iNPs is a ratedetermining step in the reduction of $4-N P$ when using these MOF@iNP composites. To further confirm this conclusion, we also studied the one-electron reduction of a bulkier molecule such as EY2- in water and in the presence of $\mathrm{NaBH}_{4}$ and the composites as catalysts. It is important to note here that, in the presence of $\mathrm{NaBH}_{4}$ but not of hollow iNPs, the initial EYspecie suffers a two-electron reduction forming the colourless specie $\mathrm{EY}^{4-}$, which can be followed by a reduction of the optical absorption at $516 \mathrm{~nm}$ corresponding to $\mathrm{EY}^{2}$. However, when both $\mathrm{NaBH}_{4}$ and hollow NPs are present, the reduction pathway changes to a one-electron reduction forming $\mathrm{EY}^{3-}$, which formation can also be followed by not only the reduction of the peak at $516 \mathrm{~nm}$ but also the appearance of a new peak at $405 \mathrm{~nm}$. ENREF $14^{14}$ 
Similar to the reduction of 4-NP, the catalytic reactions to study the reduction of $\mathrm{EY}^{2-}$ were conducted by mixing $1.5 \mathrm{~mL}$ of an aqueous solution of $\mathrm{EY}^{2-}\left(4 \times 10^{-5} \mathrm{M}\right)$ with $0.6 \mathrm{~mL}$ of an aqueous dispersion containing ZIF-8@PtNP at a Pt concentration of $2.8 \% \mathrm{w} / \mathrm{w}$ or ZIF-8@PdNP at a Pd concentration of $1.8 \% \mathrm{w} / \mathrm{w}$. Then, the volume of the resulting mixture was adjusted to $2.5 \mathrm{~mL}$ by the addition of $0.4 \mathrm{~mL}$ of deionized water. After $3 \mathrm{~min}, 0.5 \mathrm{~mL}$ of an aqueous solution of $\mathrm{NaBH}_{4}(0.12 \mathrm{M})$ was injected into the reaction mixture, and the reaction was followed every $10 \mathrm{~min}$ by UV-vis spectroscopy in the range of $300-600 \mathrm{~nm}$ (Fig. 2). For these reactions, the concentrations of $\mathrm{EY}^{2-}$ and $\mathrm{EY}^{3-}$ were determined using their previously reported extinction coefficients of $10.2 \times 10^{4} \mathrm{M}$ ${ }^{1} \cdot \mathrm{cm}^{-1}$ and $4.5 \times 10^{4} \mathrm{M}^{-1} \cdot \mathrm{cm}^{-1}, 18$ respectively. And the conversion efficiency $\left(\mathrm{EY}^{3-} / \mathrm{EY}_{0}{ }^{2-}\right)$ of each composite was measured by the ratio between the concentration of $\mathrm{EY}^{3-}$ at a given time and the initial concentration of $\mathrm{EY}^{2-}$ right after mixing all reactants.

Importantly, the general trends were very similar for both reduction reactions (Fig. 3). We found that the conversion rate $\mathrm{EY}^{3-} / \mathrm{EY}_{0}{ }^{2-}$ also increases as the ZIF-8 shell thickness decreases, and that the time at which $\mathrm{EY}^{3-}$ is starting to be formed also increases as the ZIF-8 shell thickness increases. In particular, this latter trend is more evident in the formation of $\mathrm{EY}^{3-}$. For example, in the case of ZIF-8@PtNP composites, the formation of EY3- was detected at 10 min for a thickness of $66 \pm 42 \mathrm{~nm}$; at $20 \mathrm{~min}$ for a thickness of $156 \pm 64 \mathrm{~nm}$; and at $40 \mathrm{~min}$ for a thickness of $236 \pm 64 \mathrm{~nm}$. In the case of the thicker composite, we could not detect catalytic activity even after one hour of reaction. We attributed this behaviour to the bigger size of $\mathrm{EY}^{2-}$ in comparison to $4-N P$, which makes its diffusion through the ZIF-8 shell more difficult.

In conclusion, we have determined that the MOF shell thickness is an important parameter to be considered when MOF@iNP composites are used as catalysts; as evidenced by the lower conversion efficiencies given for the composites with thicker shells at a certain time. Our findings suggest that decreasing the size (at the nanoscale) of these composites should facilitate the production of faster and more efficient catalysts. However, the smaller the composite, the lower the stability in time, which could prevent their recyclability. Nevertheless, this study clearly illustrates the importance of reporting the different size parameters (e.g. crystal size, MOF shell thickness, etc.) of MOF@iNP composites to obtain reproducible results when they are used in catalysis.

This work was supported by the Spanish MINECO (projects PN MAT2015-65354-C2-1-R and MAT2015-70725-R), the Catalan AGAUR (projects 2014-SGR-80 and 2014-SGR-612), and the ERC under the EU FP7 (ERC-Co 615954). I.I. and N.G.B. thank the MINECO for their RyC grants (RYC-2010-06530 and RYC-2012- 10991). N.G.B. also acknowledges financial support by the European Commission Seventh Framework Programme (FP7) through the Marie Curie Career Integration Grant (322153-MINE). ICN2 acknowledges the support of the Spanish MINECO through the Severo Ochoa Centres of Excellence Programme, under Grant SEV-2013-0295.

\section{Notes and references}

1. C. R. Kim, T. Uemura and S. Kitagawa, Chem. Soc. Rev., 2016, 45, 3828-3845.

2. P. Falcaro, R. Ricco, A. Yazdi, I. Imaz, S. Furukawa, D. Maspoch, R. Ameloot, J. D. Evans and C. J. Doonan, Coord. Chem. Rev., 2016, 307, Part 2, 237-254.

3. Y. Xia, H. Yang and C. T. Campbell, Acc. Chem. Res., 2013, 46, 1671-1672.

4. A. Aijaz, A. Karkamkar, Y. J. Choi, N. Tsumori, E. Ronnebro, T. Autrey, H. Shioyama and Q. Xu, J. Am. Chem. Soc., 2012, 134, 13926-13929.

5. Z. Li and H. C. Zeng, Chem. Mater., 2013, 25, 1761-1768.

6. G. Lu, S. Li, Z. Guo, O. K. Farha, B. G. Hauser, X. Qi, Y. Wang, X. Wang, S. Han, X. Liu, J. S. DuChene, H. Zhang, Q. Zhang, X. Chen, J. Ma, S. C. Loo, W. D. Wei, Y. Yang, J. T. Hupp and F. Huo, Nat Chem, 2012, 4, 310-316.

7. C. J. Stephenson, J. T. Hupp and O. K. Farha, Inorg. Chem. Front. 2015, 2, 448-452.

8. W. Zhang, Y. Liu, G. Lu, Y. Wang, S. Li, C. Cui, J. Wu, Z. Xu, D. Tian, W. Huang, J. S. DuCheneu, W. D. Wei, H. Chen, Y. Yang and F. Huo, Adv. Mater., 2015, 27, 2923-2929.

9. S. Li and F. Huo, Small, 2014, 10, 4371-4378.

$10 . \quad$ P. Hu, J. Zhuang, L. Y. Chou, H. K. Lee, X. Y. Ling, Y. C. Chuang and C. K. Tsung, J. Am. Chem. Soc., 2014, 136, 10561-10564.

11. L. Chen, Y. Peng, H. Wang, Z. Gu and C. Duan, Chem. Commun., 2014, 50, 8651-8654.

12. W. Zhang, G. Lu, C. Cui, Y. Liu, S. Li, W. Yan, C. Xing, Y. R. Chi, Y. Yang and F. Huo, Adv. Mater., 2014, 26, 4056-4060.

13. S. M. El-Sheikh, A. A. Ismail and J. F. Al-Sharab, New J. Chem., 2013, 37, 2399-2407.

14. G. Weng, M. A. Mahmoud and M. A. El-Sayed, J. Phys. Chem. C, 2012, 116, 24171-24176.

15. E. Gonzalez, F. Merkoci, R. Arenal, J. Arbiol, J. Esteve, N. G. Bastus and V. Puntes, J. Mater. Chem. A, 2016, 4, 200-208.

16. N. G. Bastús, F. Merkoçi, J. Piella and V. Puntes, Chem. Mater., 2014, 26, 2836-2846.

17. M. A. Mahmoud, B. Garlyyev and M. A. El-Sayed, J. Phys. Chem. C, 2013, 117, 21886-21893.

18. J. Zhang, L. Sun and T. Yoshida, J. Electroanal. Chem., 2011, 662, 384-395. 